\title{
Impaired regulation of NF-kB and increased susceptibility to colitis-associated tumorigenesis in CYLD-deficient mice
}

\author{
Jun Zhang, ${ }^{1}$ Brigid Stirling, ${ }^{2}$ Stephane T. Temmerman, ${ }^{1}$ Chi A. Ma, ${ }^{1}$ Ivan J. Fuss, ${ }^{1}$ \\ Jonathan M.J. Derry, ${ }^{2}$ and Ashish Jain ${ }^{1}$
}

${ }^{1}$ Laboratory of Host Defenses, National Institute of Allergy and Infectious Diseases, NIH, Bethesda, Maryland, USA. ${ }^{2}$ Amgen Inc., Seattle, Washington, USA.

\begin{abstract}
Cylindromatosis (CYLD) is a deubiquitinating enzyme that is altered in patients with familial cylindromatosis, a condition characterized by numerous benign adnexal tumors. However, the regulatory function of CYLD remains unsettled. Here we show that the development of $B$ cells, $T$ cells, and myeloid cells was unaffected in CYLD-deficient mice, but that the activation of these cells with mediators of innate and adaptive immunity resulted in enhanced NF- $K B$ and JNK activity associated with increased TNF receptor-associated factor 2 (TRAF2) and NF- $\kappa B$ essential modulator (NEMO) ubiquitination. CYLD-deficient mice were more susceptible to induced colonic inflammation and showed a dramatic increase in the incidence of tumors compared with controls in a colitis-associated cancer model. These results suggest that CYLD limits inflammation and tumorigenesis by regulating ubiquitination in vivo.
\end{abstract}

\section{Introduction}

Cylindromatosis (CYLD) is a ubiquitin-carboxyterminal hydrolase of the ovarian tumor family, which consists of enzymes that disassemble lysine 63-linked (K63-linked) polyubiquitin chains from various cellular proteins (1-4). Alterations in CYLD were first identified in patients with familial cylindromatosis, a disease characterized by numerous tumors of hair follicles and sweat glands of the head and neck (5). Genetic analysis of these skin tumors revealed that cylindromatosis patients have an inherited mutated CYLD allele and an acquired somatic mutation in the wild-type allele (6-8). The majority of mutations lead to truncated proteins that are missing the ubiquitin hydrolase domain (6).

CYLD is expressed in multiple tissues, but its precise biological function remains uncertain (9-11). Results of in vitro transfection studies suggest that CYLD downregulates NF- $\mathrm{KB}$ activity by the proteolysis of K63-linked ubiquitin from proximal NF-КB signaling constituents such as TNF receptor-associated factor 2 (TRAF2), TRAF6, and NF-kB essential modulator (NEMO; also referred to as IKK $\gamma$ ) (1-3). However, to our knowledge a direct interaction between CYLD and NEMO or the TRAFs has not previously been demonstrated in the absence of overexpression, and genetic evidence linking CYLD with NF- $\kappa B$ activity has been lacking. A recent report demonstrated that NF- $\kappa \mathrm{B}$ signaling induced by TLR4 and TNF- $\alpha$ was normal in mice deficient in CYLD (12).

To study the in vivo function of CYLD, we generated and analyzed Cyld ${ }^{-1-}$ mice. In contrast to previously published results, we report that the development of $\mathrm{T}$ and $\mathrm{B}$ lymphocytes in the Cyld ${ }^{-/-}$mice was normal. However, macrophages deficient in CYLD displayed increased NF- $\mathrm{KB}$ and JNK activity after stimulation of TLR2, TLR4, and CD40 receptors, while NF- $\kappa B$ activity after stim-

Nonstandard abbreviations used: AOM, azoxymethane; Bcl, B cell lymphoma; CAC, colitis-associated cancer; CYLD, cylindromatosis; DSS, dextran sulfate sodium; EDAR, ectodermal dysplasia receptor; NEMO, NF- $\mathrm{KB}$ essential modulator; $\mathrm{PAM}_{3} \mathrm{CSK}_{4}$, tripalmitoylated lipopeptide; RANK, receptor activator of NF-KB; RIP, receptor interacting protein; TNFR, TNF receptor; TRAF, TNF receptor-associated factor.

Conflict of interest: The authors have declared that no conflict of interest exists. Citation for this article: J. Clin. Invest. 116:3042-3049 (2006). doi:10.1172/JCI28746. ulation with TNF- $\alpha$ was normal. In a mouse model of colitis-associated cancer (CAC), we found that Cyld ${ }^{-/-}$mice developed more severe colonic inflammation and exhibited greater induction of colonic tumors. Taken together, our results provide genetic evidence that CYLD regulates NF- $\mathrm{KB}$ and JNK signaling pathways and prevents inflammation-induced tumorigenesis through its ubiquitin protease activity in vivo.

\section{Results}

Phenotypic analysis of Cyld ${ }^{-/-}$mice. Cyld ${ }^{-/-}$mice were born showing normal mendelian segregation and exhibited no overt phenotype. In initial studies, we assessed the phenotype of lymphocytes in the thymi and spleens of Cyld ${ }^{-/-}$mice. We found that the cell numbers and thymocyte subsets in the thymi of Cyld ${ }^{-/-}$mice were normal compared with those of littermate controls, as determined by analysis of CD4 and CD8 expression (Figure 1A). In addition, the spleens of Cyld ${ }^{-/}$mice contained normal numbers of $\mathrm{T}$ cell subsets as well as normal numbers of B cells compared with controls (Figure 1B). These results differ from previously published results showing that $\mathrm{Cyld}^{-/-}$mice have decreased numbers of thymic single-positive $\mathrm{T}$ cells and increased numbers of $\mathrm{B}$ cells in the spleen (12). A direct comparison of these independently generated Cyld ${ }^{-/-}$mice will likely be necessary to resolve this divergence in phenotype. Nevertheless, our findings clearly indicate that CYLD is not essential for $\mathrm{T}$ and $\mathrm{B}$ cell development.

In contrast to young mice, which appeared healthy with no overt phenotype, we observed pathologic changes in the organs of 10-month-old Cyld ${ }^{-/-}$mice, including marked lymphoid hyperplasia of the thymus and lymphoid inflammatory infiltrates in numerous tissues including the liver, spleen, lungs, and salivary glands. The liver abnormalities led to decreased serum albumin and increased serum aspartate aminotransferase levels (data not shown). These long-term changes are consistent with a role for CYLD in limiting inflammation and NF- $\mathrm{KB}$ responses in vivo.

CYLD functions as a NEMO deubiquitinase and a negative regulator of $N F-\kappa B$. To assess the function of cells lacking CYLD, we stimulated splenic B and T cells from 6- to 8-week-old Cyld ${ }^{-/-}$and con- 

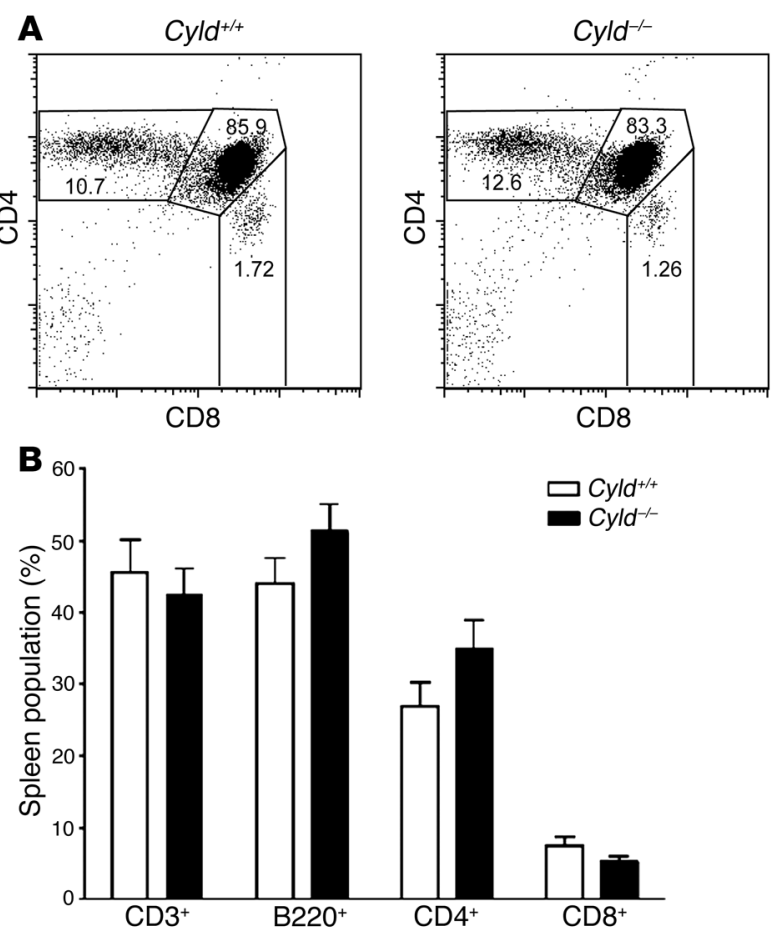

trol mice in vitro and assessed NF- $\mathrm{KB}$ DNA binding activity by EMSA. While basal NF- $\kappa \mathrm{B}$ activity was unaffected, Cyld ${ }^{-1-} \mathrm{B}$ cells exhibited elevated NF- $\kappa \mathrm{B}$ activation in response to anti-CD40 and anti-IgM stimulation (Figure 2A). In addition, enhanced NF- $\kappa \mathrm{B}$ activation was observed in Cyld ${ }^{-/-} \mathrm{T}$ cells after stimulation with anti-CD3 (Figure 2B). Recent studies have identified a critical stimulatory role for K63-linked ubiquitination of NEMO in NF- $\mathrm{KB}$ signaling induced by $\mathrm{T}$ cell receptor ligation. B cell lymphoma 10 (Bcl-10 interacts through paracaspase with the E2 ubiquitin conjugation complex (consisting of UBC13 and UEV1A) and can induce K63-linked ubiquitin conjugation of a single lysine residue (K399) in the zinc finger domain of NEMO (13). This fact, and the observation that CYLD has K63-deubiquitinating activity in vitro, prompted us to assess the ubiquitination status of endogenous NEMO in T cells. Using anti-CD3 stimulation, we observed enhanced NEMO ubiquitination in T cells from Cyld ${ }^{-/-}$ mice relative to that in control mice (Figure $2 \mathrm{C}$ ). Thus, native CYLD modulates NEMO ubiquitination in response to antigen receptor stimulation in $\mathrm{T}$ cells.

Cyld ${ }^{-1-}$ macrophages display receptor-specific enhancement of $N F-\kappa B$ activity and cytokine production. In further studies of APC function in the absence of CYLD, we determined NF- $\kappa$ B activity by EMSA in Cyld ${ }^{--}$and wild-type peritoneal macrophages after stimulation with TNF- $\alpha$, anti-CD40 Ab, and microbial components that activate TLRs, including the TLR2 agonist tripalmitoylated lipopeptide $\left(\mathrm{Pam}_{3} \mathrm{CSK}_{4}\right)$ and the TLR4 agonist LPS. Anti-CD40 Ab, $\mathrm{Pam}_{3} \mathrm{CSK}_{4}$, and LPS stimulation all resulted in enhanced NF- $\kappa \mathrm{B}$ activity in Cyld ${ }^{-/}$cells compared with wild-type controls (Figure $3 \mathrm{~A})$. In additional studies, we stimulated $\mathrm{Cyld}^{-/-}$and wild-type peritoneal macrophages with $\mathrm{Pam}_{3} \mathrm{CSK}_{4}$ plus IFN- $\gamma$, LPS plus IFN- $\gamma$, and anti-CD $40 \mathrm{Ab}$ plus IFN- $\gamma$ and measured IL- 6 and TNF- $\alpha$ secretion by ELISA. Cyld ${ }^{-/}$peritoneal macrophages synthesized greater amounts of the NF- $\kappa \mathrm{B}-$ dependent cytokines IL- 6 and TNF- $\alpha$ compared with wild-type control cells (Figure 3B).

\section{Figure 1}

$\mathrm{T}$ and $\mathrm{B}$ cell development in Cyld ${ }^{-/-}$mice. (A) Thymocytes from 4- to 6-week-old mice were analyzed for CD4 and CD8 expression. In each panel 10,000 cells were analyzed. Numbers indicate the percentage of cells in each subset. Data are representative of 4 experiments with 5 mice of each genotype. (B) Spleen populations as determined by flow cytometry from 6- to 8-week-old mice. Data are mean \pm SD calculated from 5 mice of each genotype.

In contrast, parallel studies of APC function disclosed no genotype-specific differences in NF- $\mathrm{KB}$ activation following stimulation with TNF- $\alpha$ (Figure 3A). This failure of CYLD to modulate NF- $\mathrm{B}$ activated by TNF- $\alpha$ stimulation was confirmed in vitro using an NF- $\mathrm{B}$ reporter readout and CYLD overexpression. Thus, while CYLD suppressed activation of NF- $\kappa B$ by CD40, ectodermal dysplasia receptor (EDAR), and receptor activator of NF- $\mathrm{\kappa B}$ (RANK), it had no effect on activation by TNF- $\alpha$ (Figure 3C).

TRAF2 ubiquitination and JNK activity is increased in Cyld ${ }^{-/}$cells. TNF receptor 1 (TNFR1) stimulation involves the recruitment of the TNFR1-associated protein (TRADD), TRAF2, TRAF5, and the receptor interacting protein (RIP) (14). In addition, K63 polyubiquitination of TRAF2 is a key step in such signaling. We therefore evaluated TRAF2 ubiquitination in both $\mathrm{Cyld}^{-/-}$and wild-type peritoneal macrophages stimulated with TNF- $\alpha$ and found a timedependent increase in endogenous TRAF2 ubiquitination in Cyld ${ }^{-/}$ macrophages compared with control macrophages (Figure 4A). TRAF2 ubiquitination has also been shown to mediate JNK activity; we therefore determined whether JNK activity is also enhanced in Cyld ${ }^{-/}$macrophages $(10,15,16)$. Using an anti-JNK phosphospecific $\mathrm{Ab}$, we observed that while the kinetics of JNK phosphorylation was not different between TNF- $\alpha$-stimulated Cyld ${ }^{-/}$cells and wildtype controls, the amount of phosphorylated JNK was appreciably elevated in Cyld ${ }^{-/}$cells (Figure 5A). We observed similar changes in Cyld ${ }^{--}$macrophages stimulated with LPS (Figure 5A) and in antigen receptor-stimulated Cyld ${ }^{-1}$ T cells (Figure 5B). These findings suggest that JNK activity is regulated by CYLD in response to diverse stimuli including TNF- $\alpha$.

TNF- $\alpha$-induced RIP ubiquitination in Cyld ${ }^{-/}$macrophages. Because $\mathrm{NF}-\kappa \mathrm{B}$ activation was not increased in $\mathrm{Cyld}^{-/-}$macrophages in response to TNF- $\alpha$, while the levels of ubiquitinated TRAF2 were enhanced, we next focused on RIP, a proximal mediator of NF- $\kappa B$ but not JNK signaling. TNF- $\alpha$ stimulation induces K63linked polyubiquitination of RIP, and in the absence of RIP, TNF- $\alpha$-induced NF- $\kappa B$ activity is greatly diminished $(17,18)$. To assess whether CYLD affects RIP ubiquitination, we stimulated peritoneal macrophages from $\mathrm{Cyld}^{-/-}$and wild-type mice with TNF- $\alpha$. The level of ubiquitinated RIP generated in TNF- $\alpha-$ stimulated $\mathrm{Cyld}^{-/-}$macrophages was not appreciably different from that in control macrophages (Figure 4B). These findings indicate that increased TRAF2 ubiquitination in TNF- $\alpha$-stimulated Cyld $d^{-1}$ macrophages is not associated with increased RIP ubiquitination levels, thus providing a rational explanation as to why TNF- $\alpha$-induced NF-KB signaling is not affected in Cyld ${ }^{-/-}$ mice. These results contrast with those obtained from studies of A20-1- mice, in which it has been shown that A20 downregulates TNF- $\alpha$-induced NF- $\kappa$ B responses by directly targeting RIP for K63 deubiquitination as well as for K48 ubiquitination $(19,20)$. The loss of CYLD does not affect A20 expression (A. Jain, unpublished observations), and it is likely that A20, rather than CYLD, regulates TNF- $\alpha$-induced NF- $\kappa B$ signaling. 
A
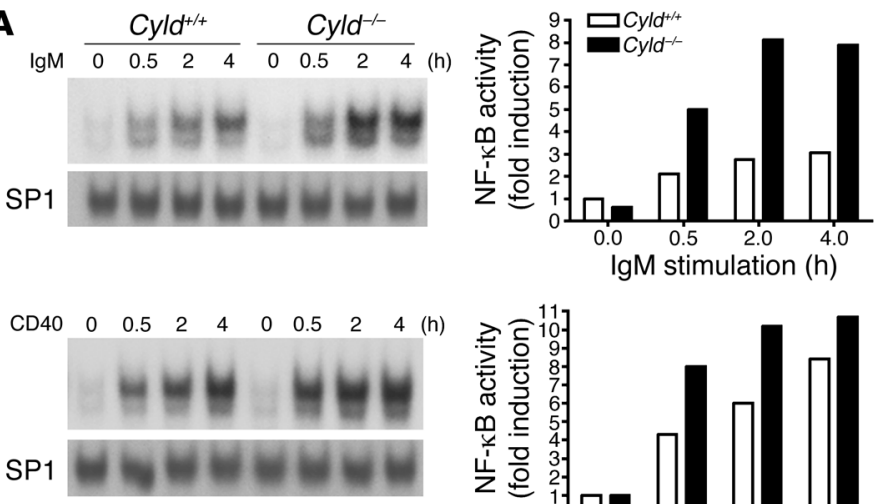

B
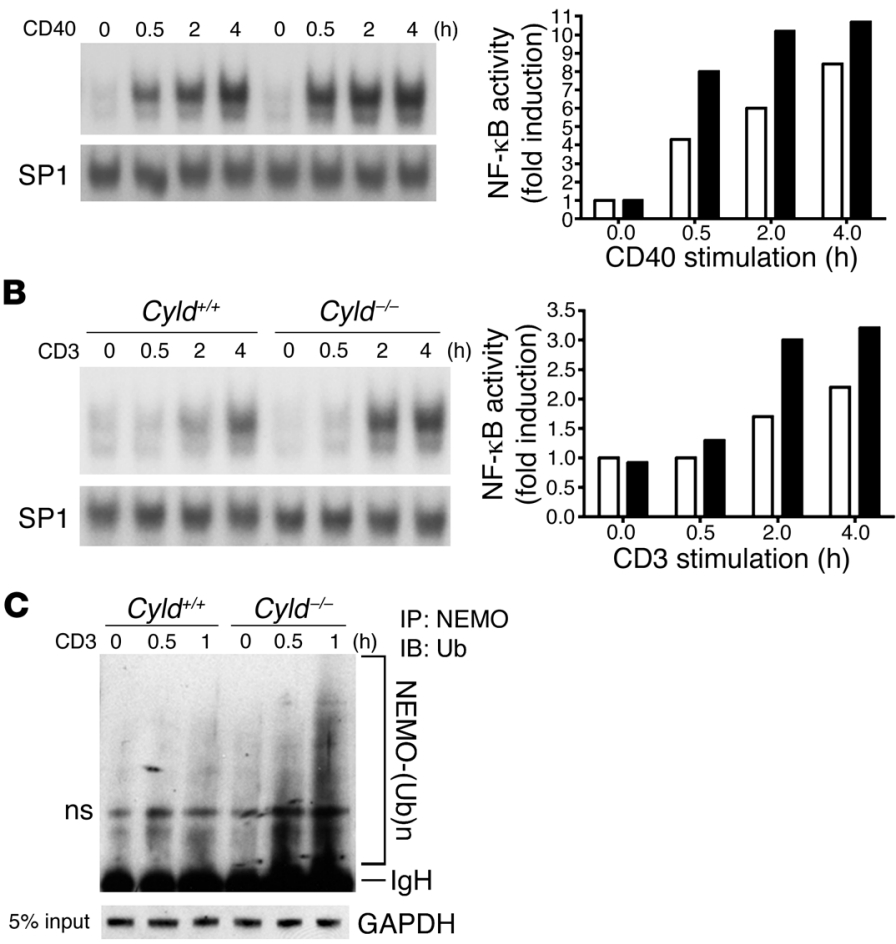

Colitis and colon tumor susceptibility in Cyld ${ }^{-/-}$mice. Our biochemical data on Cyld ${ }^{-/-}$cells provide direct evidence that CYLD is a negative regulator of NF-KB activity and suggest that $\mathrm{Cyld}^{-/-}$mice may have exaggerated inflammatory responses. To test this hypothesis, we examined the susceptibility of Cyld ${ }^{-/-}$mice to neoplasia in a CAC model. Accordingly, 6- to 8-week-old mice were injected with a single dose of azoxymethane (AOM), a DNA-alkylating agent that induces somatic mutations in colonic epithelium, followed by administration of inflammation-inducing dextran sulfate sodium (DSS) in the drinking water in triweekly cycles (Figure 6A) $(21,22)$. Such induced chronic colonic inflammation has previously been shown to increase the incidence of AOM-induced tumors (23).

In initial studies of this model, we evaluated the outcome of acute and chronic colitis in Cyld ${ }^{-/-}$and control mice. After the first DSS cycle (at 21 days of the CAC regimen), Cyld ${ }^{-/-}$mice had lost significantly more weight than the control mice (Figure 6B) and showed greater leukocyte infiltration and histologic damage, more mucosal ulcers, and increased dysplastic changes in the colonic epithelium (Table 1). Representative histologic sections are shown in Figure 6C. After the second cycle of DSS administration (at 42 days), the Cyld ${ }^{-/-}$mice exhibited greater mortality and morbidity compared with controls (Figure 6D). Throughout the duration of the experiment, Cyld ${ }^{-/-}$animals were sick and had a hunched posture, whereas control mice appeared healthy and were active. Analysis of colons at multiple time points after the administration of DSS revealed that thickening and shortening

\section{Figure 2}

CYLD is a negative regulator of NF- $\mathrm{KB}$ activation in $\mathrm{B}$ and $\mathrm{T}$ cells. (A) Cellular extracts prepared from splenic $B$ cells stimulated with anti-lgM $(10 \mu \mathrm{g} / \mathrm{ml})$ or CD40 agonist $\mathrm{Ab}(1 \mu \mathrm{g} / \mathrm{ml})$ were analyzed by EMSA for NF-KB binding. (B) Cellular extracts prepared from splenic $T$ cells and stimulated with anti-CD3 were

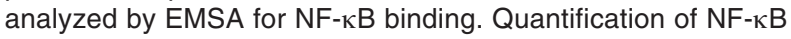
activity in $\mathbf{A}$ and $\mathbf{B}$ is represented as fold change compared with unstimulated cells (set at 1.0). Ub, ubiquitin. (C) Following T cell stimulation with anti-CD3, NEMO ubiquitination was analyzed by immunoprecipitation of the proteins from denatured cellular lysates followed by immunoblotting with an anti-ubiquitin $A b$. NEMO-(Ub)n, polyubiquitinylated NEMO; ns, nonspecific; SP1, specificity protein 1 .

of colons was more severe in the Cyld ${ }^{-/-}$mice compared with control animals, and Cyld ${ }^{-/-}$mice were frequently noted to have perforations on postmortem examination.

While both control and Cyld ${ }^{-/-}$mice treated with AOM and DSS developed colonic tumors, we observed a dramatic increase in tumor incidence in the Cyld ${ }^{-/-}$mice after the second cycle of DSS (Figure 6E). These tumors were broad-based adenocarcinomas (Figure 6F) located in the middle and distal colon, the site of inflammation induced by DSS in experimental animals. Because AOM causes mutations throughout the colon, it is likely that the more severe inflammation in the distal intestine of the Cyld ${ }^{-/-}$mice is contributing to greater tumorigenesis at this site. The number of tumors per mouse was not statistically different between control and Cyld ${ }^{-1-}$ mice after the third cycle of DSS treatment (Figure $6 \mathrm{E})$. However, at this time of analysis the majority of $\mathrm{Cyld}^{-/-}$ mice were deceased from severe colitis (Figure 6D). Thus the Cyld ${ }^{-/}$mice that survived likely experienced reduced inflammation that resulted in fewer tumors.

We next examined whether the increased occurrence of colonic tumors in the Cyld ${ }^{-/-}$mice is associated with the increased expression of NF- $\mathrm{KB}$ target genes in colonic specimens by immunohistochemistry. iNOS and COX-2 are NF- $\mathrm{\kappa B}$ genomic targets and are important regulatory molecules in inflammation and cancer development (24-26). Neither iNOS nor COX-2 expression differed significantly between the untreated wild-type and Cyld ${ }^{-/-}$ mouse colonic sections (Figure 7). However, with AOM and DSS treatment, the colonic tissue samples from $\mathrm{Cyld}^{-/-}$compared with control mice demonstrated increased staining of iNOS and COX-2 at multiple time points after DSS treatment. Taken together, these findings indicate that AOM and DSS-induced expression of iNOS and COX-2 in the colon is negatively regulated by CYLD in vivo.

\section{Discussion}

Posttranslational modification of intracellular signaling constituents through conjugation of K63-linked polyubiquitin chains has been proposed to promote the assembly of multiprotein complexes that are required for the activation of JNK and NF-кB (10, 16). There has been controversy over whether CYLD is a regulator of both of these signaling pathways $(1,2,15)$. In this report, we provide strong evidence in support of a regulatory role for CYLD in both signaling pathways by showing that TRAF2 and NEMO were hyperubiquitinated in Cyld ${ }^{-/-}$hematopoietic cells following stimulation with mediators of innate and adaptive immunity and that this was associated with increased JNK and NF- $\mathrm{KB}$ activity. In 
A
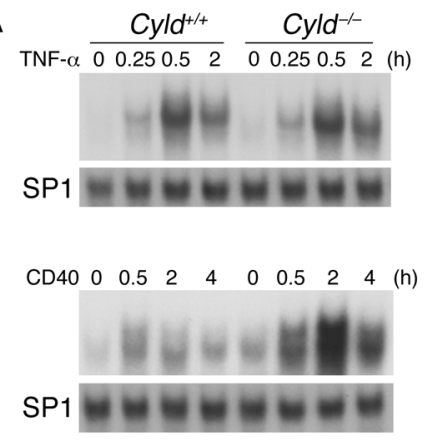

LPS $\begin{array}{lllllllll}0 & 0.5 & 1 & 2 & 0 & 0.5 & 1 & 2 & \text { (h) }\end{array}$
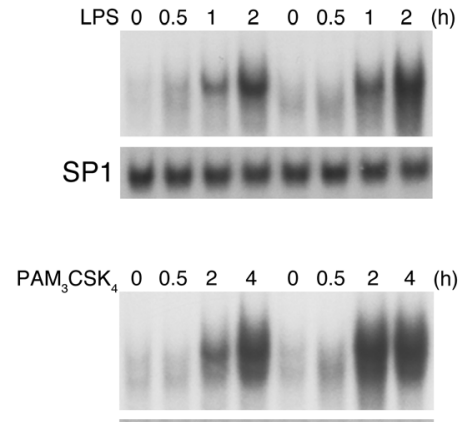

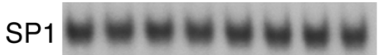
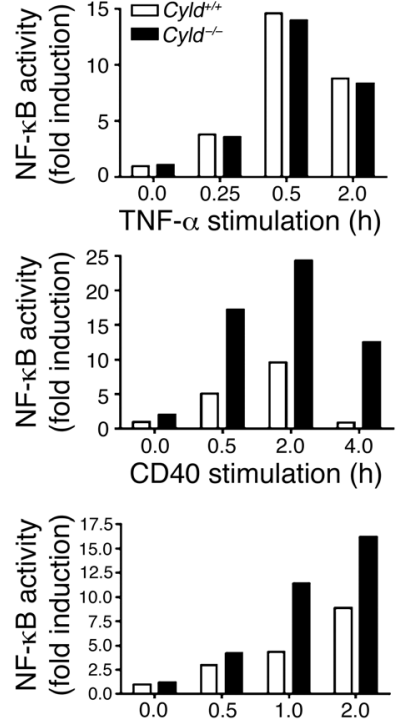

LPS stimulation (h)

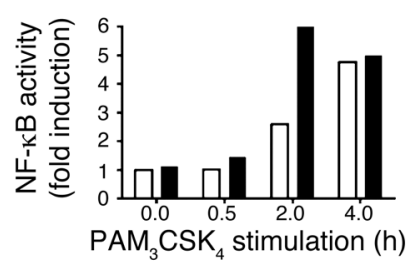

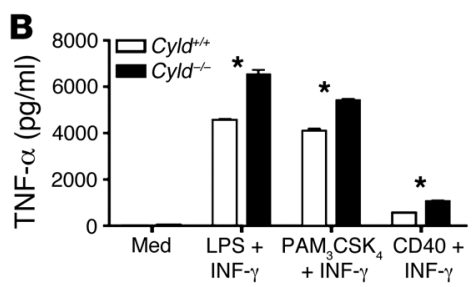
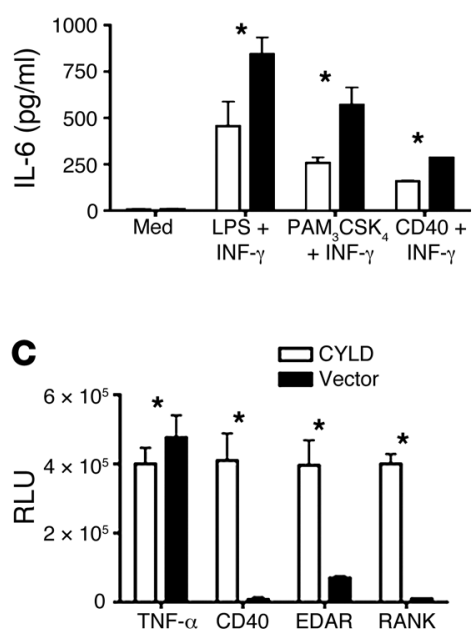

Figure 3

Comparison of triggered NF-KB activation and cytokine production in $C y / d^{-/-}$and wild-type cells. (A) Peritoneal macrophages were stimulated with TNF- $\alpha(500 \mathrm{U} / \mathrm{ml})$, CD40 agonist Ab $(1 \mu \mathrm{g} / \mathrm{ml})$, LPS $(2.5 \mu \mathrm{g} / \mathrm{ml})$, or Pam ${ }_{3} \mathrm{CSK}_{4}(150 \mathrm{ng} / \mathrm{ml})$. Cellular extracts were prepared from cells

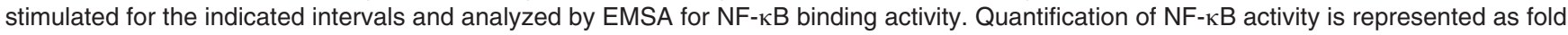
change compared with unstimulated cells (set at 1.0). (B) Peritoneal macrophages were stimulated with LPS $(2.5 \mu \mathrm{g} / \mathrm{ml}), \mathrm{Pam}_{3} \mathrm{CSK} \mathrm{K}_{4}(150 \mathrm{ng} / \mathrm{ml})$, or CD40 agonist $\mathrm{Ab}(5 \mu \mathrm{g} / \mathrm{ml})$ for 24 hours. IFN- $\gamma(10 \mathrm{ng} / \mathrm{ml})$ was added to all stimulation conditions, and the production of IL-6 and TNF- $\alpha$ into culture supernatants was assessed by ELISA. Med, medium. ${ }^{*} P<0.005, C y / d^{-/-}$versus wild-type. (C) CYLD overexpression suppresses NF-kB activation by CD40, EDAR, and RANK, but not by TNF- $\alpha$. 293M cells were transfected with an NF-kB reporter construct either in combination with a CYLD-bearing plasmid or with empty vector as a control. Cells were stimulated with TNF- $\alpha$, and luciferase activity was measured. To activate other TNFR pathways, vectors bearing CD40, EDAR, and RANK were cotransfected into 293M cells. This led to receptor overexpression and ligand-independent NF- $\mathrm{KB}$ activation caused by spontaneous receptor trimerization. Quantitation of NF- $\mathrm{KB}$ activity in the presence of CYLD is indicated as fold suppression. ${ }^{*} P<0.005, C y / d^{-/-}$versus vector.

addition, using a CAC model, we show that Cyld ${ }^{-/-}$mice were susceptible to increased intestinal inflammation and demonstrated a marked increase in the incidence of colonic tumors. These findings provide genetic evidence in support of the hypothesis that CYLD functions as a tumor suppressor gene.

In a recent study, CYLD-deficient bone marrow-derived macrophages obtained from separately generated $\mathrm{Cyld}^{-/-}$mice were reported to have normal NF-KB and JNK activity in response to TNFR1 and TLR4 stimulation (15). These data are not consistent with those reported here or with previous studies of CYLD function. Intriguingly, the same research group found that CYLD interacts with Lck, and this binding was believed to be responsible for the impaired development of single-positive thymocytes of mice deficient in CYLD. Because we did not find impairments in thymocyte development, our study indicates that CYLD is not an obligate requirement for Lck function.

While we observed increased activation of NF- $\mathrm{KB}$ and JNK ex vivo in $\mathrm{Cyld}^{-/-}$hematopoietic cells, this hyperresponsiveness did not lead to severe spontaneous inflammation. Nonetheless, we did observe lymphoid hyperplasia in aged mice. One likely explanation for this subdued phenotype is the existence of other deubiquitinating enzymes that play an important role in cellular signaling. One likely candidate is A20, an enzyme with an N-terminal ubiquitin hydrolase domain that also removes K63-linked ubiquitin from NEMO, TRAF2, and TRAF6 $(19,27)$. In contrast to CYLD, A20 also has a C-terminal ubiquitin ligase domain, which specifically targets RIP for K48 polyubiquitination and subsequent proteosomal degradation (19). A20-- mice fail to downregulate TNF- $\alpha-$ induced NF- $\mathrm{KB}$ activity and die prematurely at 6 weeks of age from chronic inflammation and cellular death (26). The phenotypic and cellular differences between the A20 and CYLD knockout mice suggest that while these 2 deubiquitinating enzymes target some of the same substrates, A20 and CYLD serve in distinct biological contexts that have yet to be fully defined.

An association between inflammation and cancer is widely appreciated, but their precise relationship remains poorly understood. Previous studies with the CAC model in mice support an indirect mechanism in which intestinal microflora stimulates inflammatory cells in the submucosa and thereby promote malignant transformation of the overlying epithelium (28-30). The role 

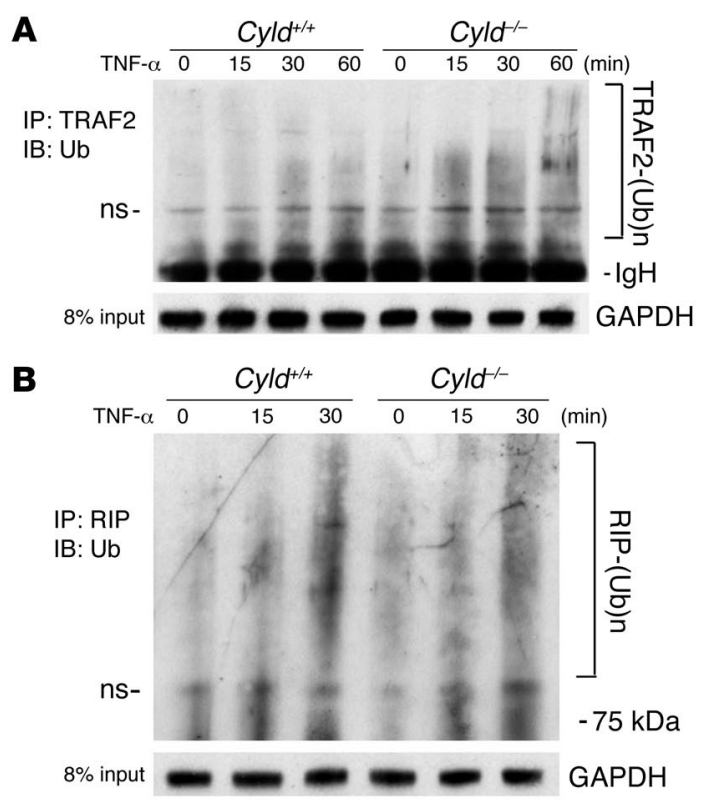

of NF- $\mathrm{KB}$ in this process was demonstrated through a conditional knockout of I- $\kappa \mathrm{B}$ kinase $\beta$ (IKK $\beta$; ref. 29). These investigators found that the incidence of colon cancer was markedly decreased in the IKK $\beta$-deleted mice, and that IKK $\beta$ had different functions in epithelial cells and myeloid cells. In epithelial cells, IKK $\beta$ was shown to regulate tumor promotion through the expression of

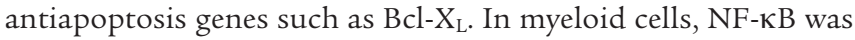
shown to regulate the expression of proinflammatory molecules that stimulated the division of genetically altered epithelial cells and thereby increase tumor size.

As shown here, stimulated Cyld ${ }^{-/-}$macrophages demonstrated increased NF- $\kappa \mathrm{B}$ activity and produced increased amounts of TNF- $\alpha$ and IL- 6 following stimulation with a variety of ligands. In addition, increased iNOS expression was observed in tumor-associated leukocytes of Cyld ${ }^{-1-}$ mice after treatment with AOM and DSS. These findings strongly suggest that CYLD regulates NF- $\mathrm{KB}$ and that the loss of this regulation contributes to the enhanced inflammation and subsequent tumor formation in Cyld ${ }^{-/-}$mice. CYLD and its known substrates such as the TRAFs and NEMO are present in epithelial cells as well. These cells are also subject

\section{Figure 5}

Comparison of cellular JNK levels in Cyld ${ }^{-1-}$ and wild-type cells. (A) Lysates prepared from peritoneal macrophages stimulated with LPS or TNF- $\alpha$ were Western blotted with phosphospecific $(\mathrm{P}-)$ antibodies recognizing activated JNKs. (B) Lysates prepared from $T$ cells stimulated with anti-CD3 were Western blotted with phosphospecific antibodies recognizing activated JNKs. SAPK, stress-activated protein kinase.

\section{Figure 4}

Comparison of TRAF2 and RIP polyubiquitination levels in $\mathrm{Cyld}^{-/-}$and wild-type cells. (A) Peritoneal macrophages stimulated with TNF- $\alpha$. TRAF2 ubiquitination was analyzed by immunoprecipitation of the proteins from denatured cellular lysates followed by immunoblotting with an anti-ubiquitin Ab. (B) Lysates prepared from peritoneal macrophages stimulated with TNF- $\alpha$ were immunoprecipitated with an RIPspecific $\mathrm{Ab}$ followed by immunoblotting with an anti-ubiquitin $\mathrm{Ab}$.

to stimulation by components of the intestinal microflora, and it is reasonable to speculate that Cyld ${ }^{-/-}$epithelial cells would show enhanced survival characteristics as a consequence of enhanced TLR signaling. This notion is supported by the finding that after AOM and DSS treatment, the Cyld ${ }^{-/-}$epithelial cells had increased expression of COX-2, an NF-кB-regulated protein involved in cellular proliferation and antiapoptotic activity. As a consequence, Cyld ${ }^{-/}$epithelial cells subsequently accumulated additional oncogenic mutations that ultimately led to enhanced tumorigenesis. These findings suggest that modest differences in NF- $\kappa \mathrm{B}$ activity and cytokine production occurring in the absence of CYLD lead to marked differences in the development of induced inflammation-associated cancer.

Recently, mice deficient in CYLD were also shown to be more susceptible to skin tumors induced by 7, 12-dimethylbenz(a)anth racene (DMBA) and 12-O-tetradecanoylphorbol-13-acetate (TPA) than were wild-type mice (31). These authors linked the loss of CYLD in keratinocytes to increased $\mathrm{Bcl}-3$ polyubiquitination and increased transcription of Bcl-3-dependant target genes. Therefore, the mechanism of CYLD-mediated NF- $\mathrm{BB}$ suppression may vary in different tumors.

In summary, our study provides genetic evidence that CYLD plays a regulatory role in the function of intracellular signaling proteins of the hematopoietic system and that its deficiency leads to increased susceptibility to tumor induction. It is noteworthy, however, that such susceptibility was only apparent in the presence of
A
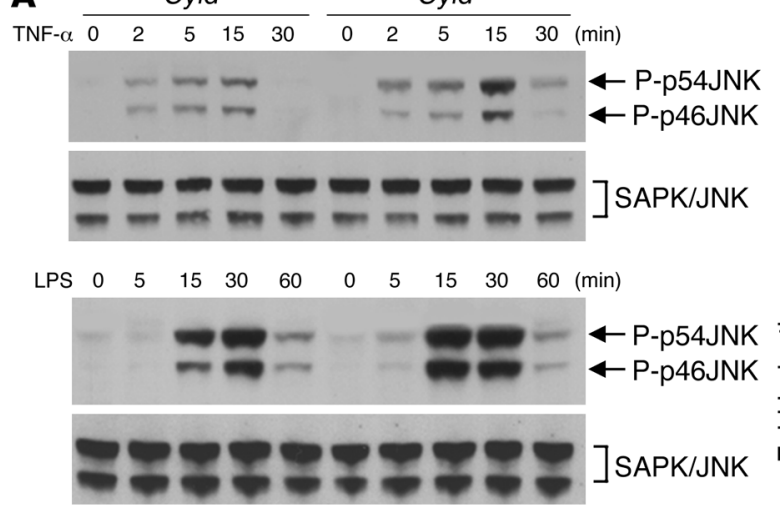

B

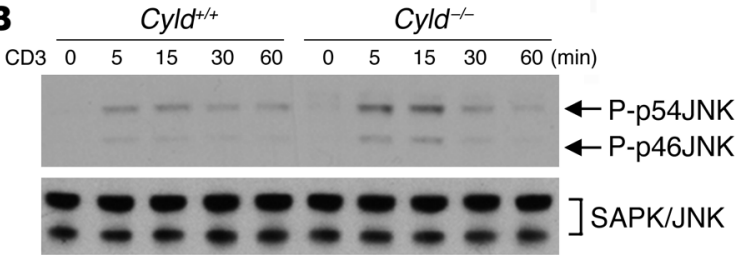

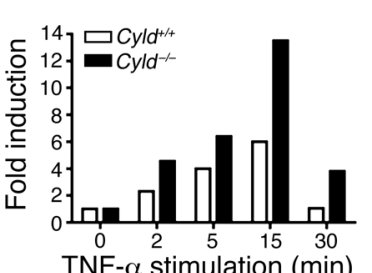

TNF- $\alpha$ stimulation (min)
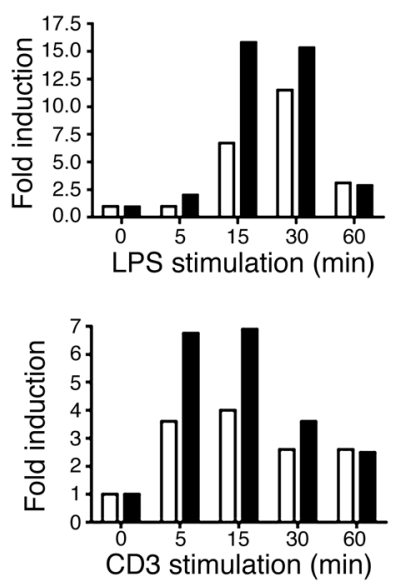
A
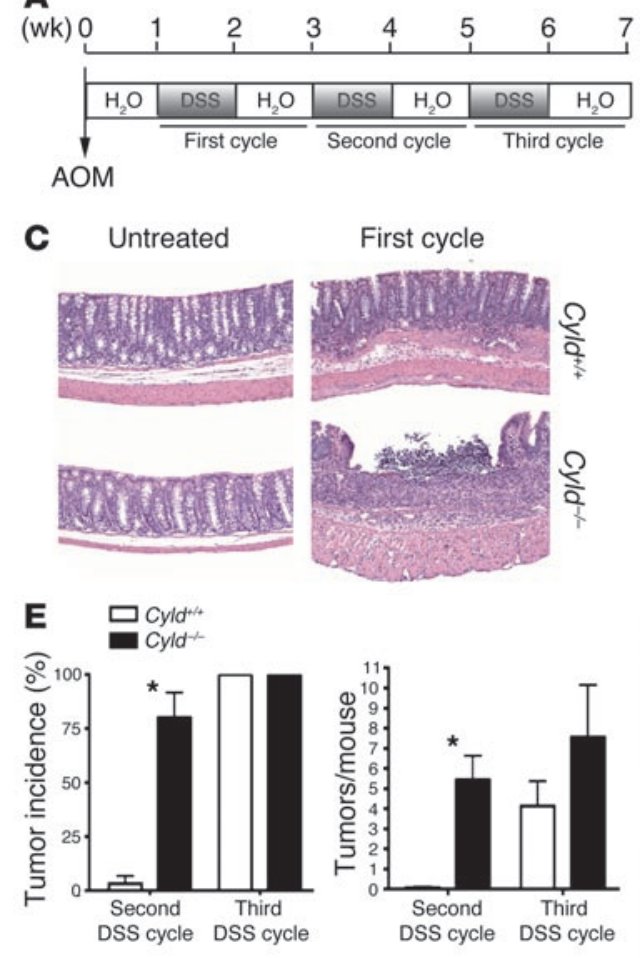

First cycle

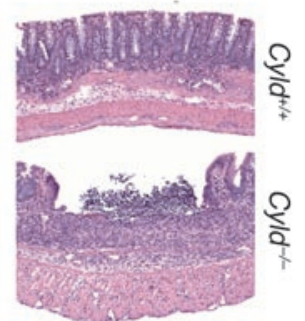

B

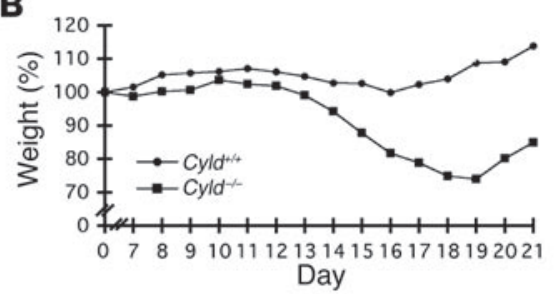

D

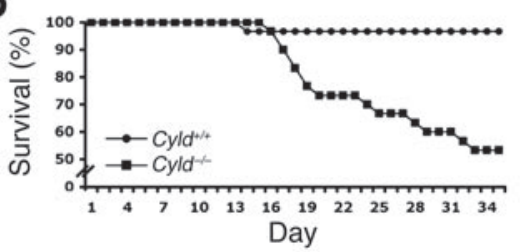

F

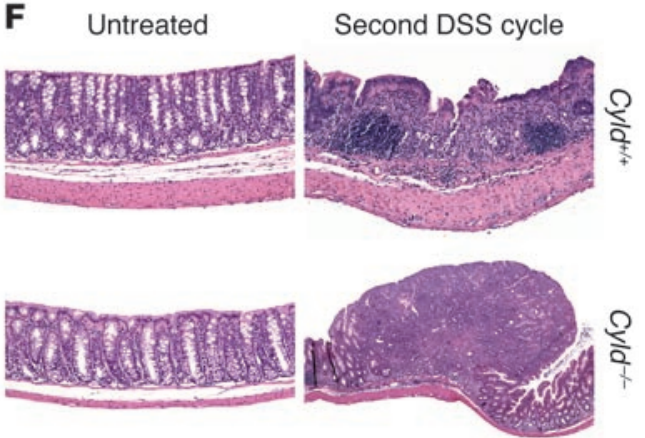

\section{Figure 6}

Increased colonic inflammation and tumor development in $\mathrm{Cy}^{-1 /-}$ mice. (A) Schematic of the AOM and DSS CAC model. (B) Weight loss during the first cycle of DSS treatment in $\mathrm{Cyld}^{-/-}$and wild-type mice, expressed as percent of the starting weight for each cohort. (C) Histologies of $\mathrm{Cyld}^{-1-}$ and wild-type colons, either left untreated or 7 days after the first cycle of DSS treatment. Magnification, $\times 50$. (D) Survival curves of $\mathrm{Cyld}^{-1-}$ and wild-type mice ( $n=60$ per group) injected with AOM followed by DSS. Curves are statistically different $(P<0.001)$. (E) Incidence and total number of tumors $(>0.5 \mathrm{~mm})$ in the colons of $\mathrm{Cyld}^{-/-}$and wild-type mice after administration of AOM followed by DSS. Mice were sacrificed 7 days after the first (left) or the second cycle (right) of DSS treatment. ${ }^{*} P<0.001$; Cyld ${ }^{-1-}$ versus wild-type. (F) Typical colon histologies demonstrating broad-based adenocarcinoma in a Cyld ${ }^{-/-}$section 7 days after the second cycle of DSS treatment. Magnification, $\times 50$. inflammation, emphasizing that regulatory abnormalities seen in the absence of CYLD are mainly observed in hyperactivated cells.

\section{Methods}

Generation of Cyld ${ }^{-1}$ mice. All mice were maintained in the National Institute of Allergy and Infectious Diseases (NIAID) animal holding facilities. Animal use adhered to NIH laboratory animal care guidelines and all animal experiments were approved by the NIAID Animal Care and Use Committee review board. Cyld $d^{-1}$ mice were generated by Lexicon Genetics Inc. The ATG start codon is in exon 2, and a gene-targeting construct was designed to delete exons 2 and 3 and replace them with a lac $Z$ reporter and a neomycin resistance gene (Supplemental Figure 1; supplemental material available online with this article; doi:10.1172/JCI28746DS1). The targeting vector was linearized and electroporated into Lex-1 ES cells. Clones resistant to G417 were selected and screened for homologous recombinants by Southern blot analysis. Two targeted ES cell clones were microinjected into C57BL/6 (albino) blastocysts, and the resulting chimeras were mated to C57BL/6 (albino) females to generate mice heterozygous for the cyld mutation. Homozygous knockout animals were obtained by mating of heterozygous males and females. Genotyping was performed by PCR on tail-derived genomic DNA, and germline transmission was confirmed by Southern blot analysis.

Fluorescence-activated cell sorting analysis. Cells were obtained from thymus, spleen, lymph node, and blood using standard methods and stained with combinations of the following antibodies to distinguish hematopoietic subsets: CD3, CD4, CD8, IgM, IgD, B220, CD11c, CD11b, CD25, CD69, CD19, and 2B4. Samples were analyzed using a FACScan (BD Biosciences) with CellQuest software version 2 (BD Biosciences).

Reporter gene assays. We seeded $2 \times 10^{5} 293 \mathrm{M}$ cells into 6 -well plates and, after overnight incubation, transfected them with Lipofectamine 2000 (Invitrogen) with $0.5 \mu \mathrm{g}$ of reporter plasmids in combination with different concentrations of CD40-, RANK-, or EDAR-expressing plasmids.
Cells were stimulated with TNF- $\alpha(10 \mathrm{ng} / \mathrm{ml}) 24$ hours after transfection. Corresponding empty vectors were added into all transfections to ensure a consistent $2 \mu \mathrm{g}$ of total DNA per transfection. Twenty-four hours after transfection, lysates were prepared, and luciferase activity was measured using the Dual-Luciferase reporter assay system (Promega).

ELISA. Peritoneal cavity macrophages were harvested with $10 \mathrm{ml}$ cold PBS 72 hours after 3\% thioglycollate broth injection and washed twice with DMEM. Cells were resuspended in DMEM with 10\% FBS and 1\% penicillin-streptomycin and stimulated for 24 hours with LPS (InvivoGen) plus IFN- $\gamma$ (R\&D Systems), Pam $_{3} \mathrm{CSK}_{4}$ (InvivoGen) plus IFN- $\gamma$, or anti-CD40 $\mathrm{Ab}$ (BD Biosciences - Pharmingen) plus IFN- $\gamma$. Culture supernatants were harvested, and cytokine concentrations were measured by R\&D Systems Duo Set kits. In brief, the supernatants were applied onto plastic plates

\section{Table 1}

Histopathologic scoring of colons in Cyld ${ }^{-/-}$and $C y / d^{+/+}$mice following DSS administration

$\begin{array}{lcc} & \text { Cyld }{ }^{+/+} & \text {Cyld } \text { d- }^{--} \\ \text {Epithelial injury } & + & +++ \\ \text { Ulcer/erosion } & 0 \text { to }+ & +++ \\ \text { Infiltrating leukocyte } & + \text { to }++ & +++ \text { to }++++ \\ \text { Colonic dysplasia } & \text { Low-grade } & \text { High-grade }\end{array}$

Tissue sections were obtained from mice 7 days after the first cycle of DSS administration. + , minimal ( $<25 \%$ of section involvement); ++ , mild $(<50 \%$ of section involvement); +++ , moderate $(<75 \%$ of section involvement); ++++, severe ( $<100 \%$ of section involvement). Low-grade dysplasia is defined as mild dysplastic changes with decreased goblet cells extending to the surface epithelium. In high-grade dysplasia, a greater degree of cytologic atypia is evident: the nuclei are enlarged and have irregular contours, and a loss of normal polarity is observed (31-33). 

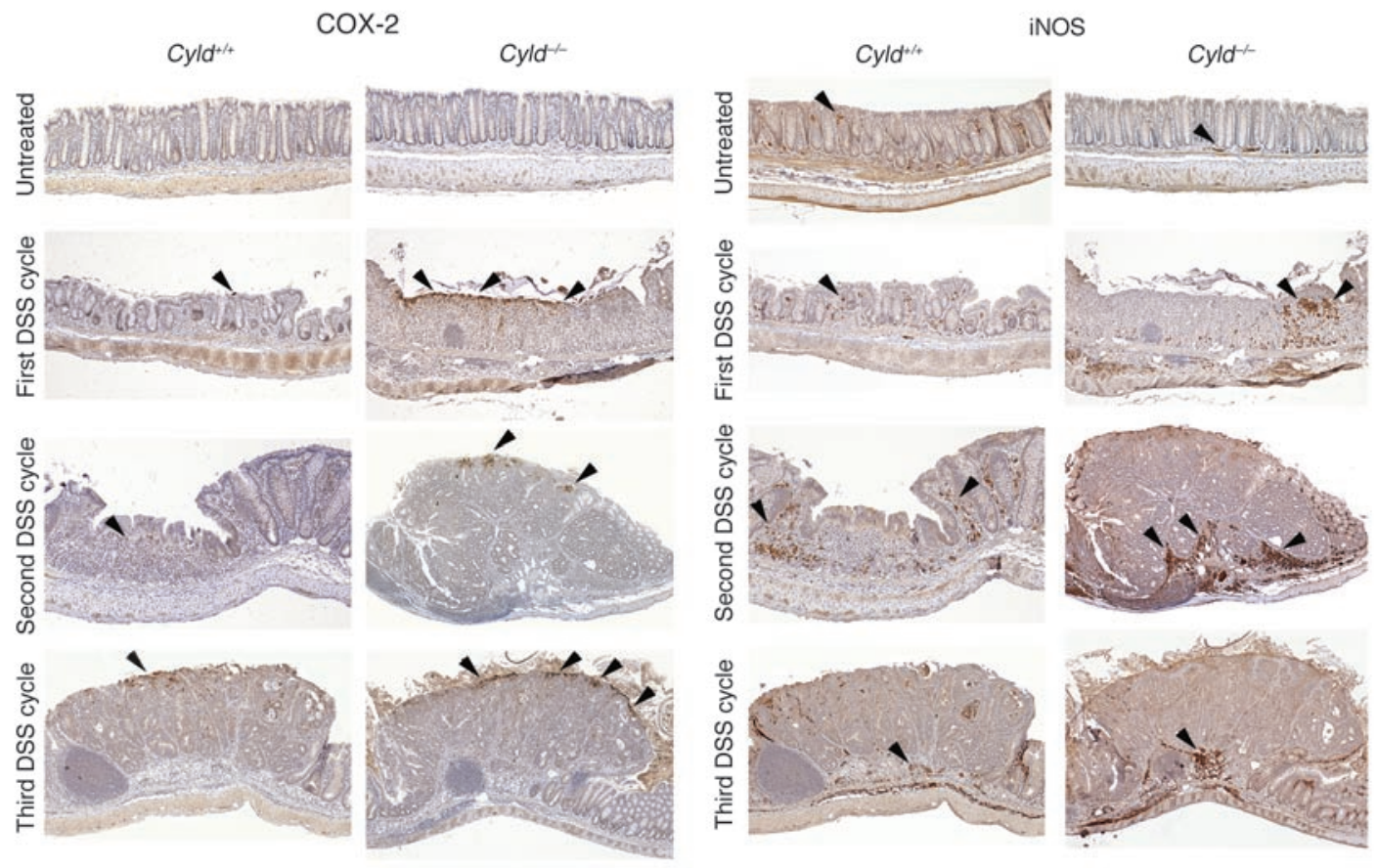

\section{Figure 7}

COX-2 and iNOS immunostaining of tissue sections obtained from wild-type and Cyld ${ }^{-1-}$ mice 7 days after the first, second, and third cycles of DSS treatment. Numerous COX-2-positive cells were observed in the epithelium, and iNOS-positive cells were observed within broadly based inflammatory infiltrating cells, of the adenomatous tissue of $C y / d^{-1-}$ mice. Arrowheads indicate positively stained cells. Magnification, $\times 50$.

precoated with anti-IL- $6 \mathrm{mAb}$ or anti-TNF- $\alpha \mathrm{mAb}$. After washing, plates were probed with biotin-conjugated detection $\mathrm{Ab}$ and HRP-conjugated streptavidin and developed with TMB1 component HRP microwell substrate (BioFx Laboratories). Absorbance at $450 \mathrm{~nm}$ was measured by spectrophotometer (Kinetic Microplate reader; Molecular Devices).

EMSA. Spleen B cells and CD4 T cells were isolated using CD45R (B220) and CD4 (L3T4) microbeads (Miltenyi Biotec), respectively. Macrophages were harvested from mouse peritoneal cavities 3 days after injection of $3 \%$ (wt/vol) thioglycolate (Sigma-Aldrich) broth. B cells were stimulated with anti-CD40 and anti-IgM Abs (BD Biosciences - Pharmingen). CD4 T cells were stimulated with plate-coated anti-CD3 Ab $(10 \mu \mathrm{g}$; BD Biosciences - Pharmingen). Macrophages were stimulated with LPS $(2.5 \mu \mathrm{g} / \mathrm{ml})$, antiCD40 $(1 \mu \mathrm{g} / \mathrm{ml})$, Pam $_{3} \mathrm{CSK}_{4}(150 \mathrm{ng} / \mathrm{ml})$, or recombinant murine TNF- $\alpha$ $(500 \mathrm{U} / \mathrm{ml})$ at different time points. Whole-cell extracts from untreated or stimulated B cells, CD4 T cells, and macrophages were prepared using whole-cell lysis buffer (50 mM Tris- $\mathrm{HCl}$, pH 7.5; $100 \mathrm{mM} \mathrm{NaCl} ; 50 \mathrm{mM} \mathrm{NaF}$; $0.1 \mathrm{mM} \mathrm{Na}_{3} \mathrm{VO}_{4} ; 30 \mathrm{mM}$ sodium pyrophosphate; $0.5 \% \mathrm{NP} 40 ; 0.6 \%$ diisopropyl fluorophosphate; and complete protease inhibitor [Roche Diagnostics]). Protein concentrations were determined using a Micro BCATM Protein Assay Kit (Pierce). Binding reactions for NF-אB were preformed using the Trans-AM NF- $\kappa B$ Kit (Active Motif) according to the manufacturer's instructions. Cellular lysates were incubated with ${ }^{32} \mathrm{P}$-labeled double-stranded NF-кB oligonucleotide (5'-AGTTGAGGGGACTTTCCCAGGC-3') for 20 minutes before electrophoresis on $5 \%$ nondenaturing polyacrylamide gels.

IP, ubiquitination assay, and Western blotting. Cells were washed with PBS before being lysed in IP buffer $(50 \mathrm{mM}$ Tris-Cl, $\mathrm{pH} 7.5 ; 150 \mathrm{mM} \mathrm{NaCl}$; 3 mM EGTA; 1\% Triton-X 100; 0.5\% NP-40; 10 mM N-ethylaleimide [Sigma-Aldrich]; $1 \mathrm{mM}$ sodium orthovanadate [Sigma-Aldrich]; $10 \%$ glycerol [Invitrogen]; and protease inhibitor mixture with EDTA [Roche Diagnostics]). Cellular lysates were heat denatured with $1 \%$ SDS, diluted 1:10, precleared with Protein A/G Plus agarose beads (Santa Cruz Biotechnol- ogy Inc.), and incubated with mouse mAb against NEMO, TRAF2, or RIP (BD Biosciences - Pharmingen) followed by incubation with Protein A/G Plus beads. IPs were washed with IP buffer followed by Tris-buffered saline, heat denatured in NuPAGE-LDS sample buffer (Invitrogen), and resolved in a $4 \%-12 \%$ gradient NuPAGE Bis-Tris polyacrylamide gel. Proteins were transferred to Hybond-ECL nitrocellulose membrane (Amersham Biosciences) using a Trans-Blot SD apparatus (Bio-Rad) and probed with rabbit anti-ubiquitin antibodies (Santa Cruz Biotechnology Inc. and Imgenex). In $\mathrm{T}$ cell experiments, $\mathrm{CD} 4^{+} \mathrm{T}$ cells were stimulated with plate-coated antiCD3 (10 $\mu \mathrm{g}$; BD Biosciences - Pharmingen) for 24 hours and then expanded in IL-2 for 7 days before use. The anti-CYLD Ab was a gift of S.C. Sun (Pennsylvania State University, Hershey, Pennsylvania, USA).

Tumor induction and analysis. Cyld ${ }^{-/-}$mice (6-8 weeks old; C57BL/6 × 129 mixed background) and control B6129SF 2 mice (The Jackson Laboratory) were injected i.p. with $10 \mathrm{mg} / \mathrm{kg} \mathrm{AOM}$ (National Cancer Institute). After 1 week, 3\% DSS (MW, 36-50 kDa; MP Biomedicals) was given in the drinking water for 1 week, followed by 1 week of regular water. This cycle was repeated twice ( 1 week of $3 \%$ DSS and 1 week of regular water), and mice were sacrificed at the end of each cycle. Colons were removed, flushed with PBS, fixed using the "Swiss-roll" technique in $4 \%$ paraformaldehyde at $4{ }^{\circ} \mathrm{C}$ overnight, and paraffin-embedded. Sections $(5 \mu \mathrm{m})$ were cut stepwise $(200 \mu \mathrm{m})$ through the complete block and stained with H\&E. Tumor counts and colitis disease scores were performed in a blinded fashion. Paraffin block sections were immunostained with an anti-iNOS Ab (BD Biosciences) or with anti-COX-2 (Cayman Chemical). Appropriate control material (according to the manufacturer's instructions) was used for each run. For negative controls, the primary Ab was omitted. After immunohistochemistry, the samples were assessed by light microscopy.

Statistics. Two-tailed Student's $t$ test was used to evaluate statistical significance. Analysis was performed using Prism 4 (GraphPad Software). $P$ values less than 0.05 were considered to be significant. 


\section{Acknowledgments}

We wish to thank Uli Seibenlist, Warren Strober, Phil Murphy, Gary Means, and Jacques Peschon for helpful discussions; Michelle Pace, Sue Kanaly, Dan Branstetter, Miriam Anver, and Matthew Starost for pathology support; and Keith Charrier for FACS analysis.

1. Kovalenko, A., et al. 2003. The tumour suppressor CYLD negatively regulates NF-kappaB signalling by deubiquitination. Nature. 424:801-805.

2. Trompouki, E., et al. 2003. CYLD is a deubiquitinating enzyme that negatively regulates NFkappaB activation by TNFR family members. Nature. 424:793-796.

3. Brummelkamp, T.R., Nijman, S.M., Dirac, A.M., and Bernards, R. 2003. Loss of the cylindromatosis tumour suppressor inhibits apoptosis by activating NF-kappaB. Nature. 424:797-801.

4. Makarova, K.S., Aravind, L., and Koonin, E.V. 2000. A novel superfamily of predicted cysteine proteases from eukaryotes, viruses and Chlamydia pneumoniae. Trends Biochem. Sci. 25:50-52.

5. Brooke, H. 1892. Epithelioma adenoides cysticum. Br. J. Dermatol. 4:269-287.

6. Bignell, G.R., et al. 2000. Identification of the familial cylindromatosis tumour-suppressor gene. Nat. Genet. 25:160-165.

7. Lian, F., and Cockerell, C.J. 2005. Cutaneous appendage tumors: familial cylindromatosis and associated tumors update. Adv. Dermatol. 21:217-234.

8. Biggs, P.J., et al. 1995. Familial cylindromatosis (turban tumour syndrome) gene localised to chromosome 16q12-q13: evidence for its role as a tumour suppressor gene. Nat. Genet. 11:441-443.

9. Ben-Neriah, Y. 2002. Regulatory functions of ubiquitination in the immune system. Nat. Immunol. 3:20-26.

10. Chen, Z.J. 2005. Ubiquitin signalling in the NFkappaB pathway. Nat. Cell Biol. 7:758-765.

11. Deng, L., et al. 2000. Activation of the IkappaB kinase complex by TRAF6 requires a dimeric ubiquitin-conjugating enzyme complex and a unique polyubiquitin chain. Cell. 103:351-361.

Received for publication April 5, 2006, and accepted in revised form September 12, 2006.

Address correspondence to: Ashish Jain, NIH, 10 Center Drive, Room 11N228, Bethesda, Maryland 20892, USA. Phone: (301) 594-5691; Fax: (301) 402-2240; E-mail: ajain@niaid.nih.gov.

12. Reiley, W.W., et al. 2006. Regulation of T cell development by the deubiquitinating enzyme CYLD. Nat. Immunol. 7:369-370.

13. Zhou, H., et al. 2004. Bcl10 activates the NF-kap$\mathrm{paB}$ pathway through ubiquitination of NEMO. Nature. 427:167-171.

14. Chen, G., and Goeddel, D.V. 2002. TNF-R1 signaling: a beautiful pathway. Science. 296:1634-1635.

15. Reiley, W., Zhang, M., and Sun, S.C. 2004. Negative regulation of JNK signaling by the tumor suppressor CYLD. J. Biol. Chem. 279:55161-55167.

16. Habelhah, H., et al. 2004. Ubiquitination and translocation of TRAF2 is required for activation of JNK but not of p38 or NF-kappaB. EMBO J. 23:322-332.

17. Ting, A.T., Pimentel-Muinos, F.X., and Seed, B. 1996. RIP mediates tumor necrosis factor receptor 1 activation of NF-kappaB but not Fas/APO-1-initiated apoptosis. EMBO J. 15:6189-6196.

18. Kelliher, M.A., et al. 1998. The death domain kinase RIP mediates the TNF-induced NF-kappaB signal. Immunity. 8:297-303.

19. Wertz, I.E., et al. 2004. De-ubiquitination and ubiquitin ligase domains of A20 downregulate NF-kappaB signalling. Nature. 430:694-699.

20. Legler, D.F., Micheau, O., Doucey, M.A., Tschopp, J., and Bron, C. 2003. Recruitment of TNF receptor 1 to lipid rafts is essential for TNFalpha-mediated NF-kappaB activation. Immunity. 18:655-664.

21. Boivin, G.P., et al. 2003. Pathology of mouse models of intestinal cancer: consensus report and recommendations. Gastroenterology. 124:762-777.

22. Pegg, A.E. 1984. Methylation of the O6 position of guanine in DNA is the most likely initiating event in carcinogenesis by methylating agents. Cancer Invest. 2:223-231.

23. Okayasu, I., Ohkusa, T., Kajiura, K., Kanno, J., and
Sakamoto, S. 1996. Promotion of colorectal neoplasia in experimental murine ulcerative colitis. Gut. 39:87-92.

24. Peskin, A.V. 1997. Interaction of reactive oxygen species with DNA. A review. Biochemistry (Mosc.). 62:1341-1347.

25. Lu, H., Ouyang, W., and Huang, C. 2006. Inflammation, a key event in cancer development. Mol. Cancer Res. 4:221-233.

26. Li, Q., and Verma, I.M. 2002. NF-kappaB regulation in the immune system. Nat. Rev. Immunol. 2:725-734.

27. Boone, D.L., et al. 2004. The ubiquitin-modifying enzyme A20 is required for termination of Toll-like receptor responses. Nat. Immunol. 5:1052-1060.

28. Clevers, H. 2004. At the crossroads of inflammation and cancer. Cell. 118:671-674.

29. Greten, F.R., et al. 2004. IKKbeta links inflammation and tumorigenesis in a mouse model of colitis-associated cancer. Cell. 118:285-296.

30. Rakoff-Nahoum, S., Paglino, J., Eslami-Varzaneh, F., Edberg, S., and Medzhitov, R. 2004. Recognition of commensal microflora by toll-like receptors is required for intestinal homeostasis. Cell. 118:229-241.

31. Massoumi, R., Chmielarska, K., Hennecke, K., Pfeifer, A., and Fassler, R. 2006. Cyld inhibits tumor cell proliferation by blocking Bcl-3-dependent NF-kappaB signaling. Cell. 125:665-677.

32. Pascal, R.R. 1994. Dysplasia and early carcinoma in inflammatory bowel disease and colorectal adenomas. Hum. Pathol. 25:1160-1171.

33. Riddell, R.H., et al. 1983. Dysplasia in inflammatory bowel disease: standardized classification with provisional clinical applications. Hum. Pathol. 14:931-968. 